BULL. AUSTRAL. MATH. SOC.

VOL. $22(1980), 211-217$.

\title{
A NOTE ON THE CONCEPT OF MULTISET
}

\author{
J.L, HICKMAN
}

\begin{abstract}
In various papers on some of the theoretical aspects of computing, the reader is confronted with a mathematical entity called a "multiset", and is told that a multiset is "an unordered collection of elements that may have repeated occurrences of identical elements" (see, for example, Nachum Dershowitz and Zohar Manna, "Proving termination with multiset orderings", Lecture Notes in Computer Science, 71). These entities are then manipulated in accordance with the classical laws of set algebra. The purpose of this note is to present a formal foundation for this concept of multiset, and to show by means of examples that if this foundation is adopted then, although certain sections of classical set algebra can be applied to multisets, it certainly cannot be applied in total.
\end{abstract}

We are told by various authors of papers on computing that a multiset is an unordered collection of elements that may have repeated occurrences of identical elements. Then, sometimes without further ado, we are confronted with various formulae that look like formulae from classical set algebra, except that the operands are not sets but multisets. An illustration is afforded by [1], in which, immediately after being presented with the above definition, we invited to form the compound $M-X \cup Y$, where $M, X, Y$ are multisets with $\emptyset \neq X \subseteq M$. At no stage are we told what "-", "U" and "ᄃ" stand for.

Received 10 March 1980. The work contained in this paper was done whilst the author was a Research Officer at the Australian National University . 
Clearly one cannot expect a paper such as [1], in which multisets are used merely as a tool, to contain a fully fledged formal theory. But, in view of the apparent lack of any readily available standard treatment of the topic, we suggest, with due respect, that those writers who wish to use multisets might present the reader with some fundamental definitions, or at least warn him that any analogy between multiset operations and set operations cannot be pushed too far.

In formulating the definitions contained in this note, we have been guided by two principles. Firstly, the principle that in any multiset one should be able to say exactly how many times a particular element appears, but not be able to "pick out" any one copy of a given element in preference to any other copy (for otherwise the two copies involved would not be "identical"). For example, if $f$ is a mapping between two multisets and $x, y$ are two copies of a given element in the domain of $f$, then $f(x)$, $f(y)$ must be either equal or else copies of a given element in the range of $f$. The second principle is that if one sets up a formal theory after some results in the field have already been obtained, then one should make every effort to have the theory fit the results, for clearly this is how people want the concepts to work. In this effort we have taken into consideration, in addition to those practical papers that we could find, the list of intuitive multiset properties given by Knuth in [2] (although we cannot entirely agree with his subsequent comments upon the construction of a formal theory of multisets). We shall see later that it is very difficult to define the complementation operator - in any way which preserves our intuitive notions of what this operator should do, and it is perhaps significant that Knuth, in contrast to Dershowitz and Manna, eschews complementation of multisets entirely.

The set-theoretical background will be Zermelo-Fraenkel with the Axiom of Choice. It is well-known that within such a system one can define a unary predicate $\mathcal{C}$ with $\mathcal{C}(x)$ having the intuitive meaning, " $x$ is a cardinal". Given any function $f$, we use "Dom $(f)$ ", "Ran $(f)$ " to denote its domain and range respectively.

DEFINITION 1. A multiset (m-set) $M$ is a function such that for each $x \in \operatorname{Dom}(M)$ we have $C(M(x))$ and $M(x) \neq 0$. We define a binary predicate $E$ by taking $x E M$ to mean $M$ is an $m$-set and $x \in \operatorname{Dom}(M)$ ". 
The idea is of course obvious; if $M$ is an $m$-set, and $x E M$, then $M$ is supposed to "contain" $M(x)$ copies of $x$ (including $x$ itself). This suggests very strongly that the collection of all m-sets $M$ such that $M(x)=1$ for each $x E M$ should be structurally equivalent to the class of all sets. This equivalence will be seen to hold in the sense that each definition that we shall make will, when restricted to this special subcollection of $m$-sets, be logically equivalent to some classical definition in set theory. In view of this we shall take the convenient step of informally identifying a set with the corresponding "unit" $m$-set.

DEFINITION 2. Let $M, N$ be m-sets. We say that $M$ is an $m$-subset of $N\left(M \subseteq_{m} N\right)$ if for each $K E M$ we have $K E N$ and $M(K) \leq N(K)$, where " $\leq "$ is the usual cardinal ordering. If $\operatorname{Dom}(M)=\operatorname{Dom}(N)$ then we say that $M$ is a full m-subset of $N$. For any $m$-set $N$, we define its powerset $P(N)$ to be the set of all m-subsets of $N$.

We note that according to this definition, $P(N)$ is always an ordinary set; we could see no good reason for introducing repeated elements into a powerset. However, as we shall see later, this does mean that one of the cornerstones of classical set theory, Cantor's powerset theorem, fails for multisets.

DEFINITION 3. Let $M, N$ be m-sets. We define an m-map $s: M \rightarrow N$ to be a function $\operatorname{Dom}(M) \rightarrow \operatorname{Dom}(N)$. We say that $s$ is m-injective if $s$ is injective and $M(K) \leq N(s(K))$ for each $K E M$, and that $s$ is $m$-surjective if $s$ is surjective and $M(K) \geq N(s(K))$ for each $K E M$. We say that $s$ is $m$-bijective if $s$ is m-injective and $m$-surjective.

This at first sight might seem an unnecessarily restrictive definition of an m-map, for $m$-sets are supposed to be richer objects in some sense than ordinary sets, and one might expect that this extra richness would be reflected in the definition of an m-map. But as we explained previously, we do not feel that one should be allowed to map identical elements to nonidentical elements: furthermore, the above definition does seem to reflect the needs of those who use multisets. Hence it is in accordance with our two guiding principles.

DEFINITION 4 . Let $X$ be an ordinary set whose elements are m-sets. 
(a) We define $U X$ by $\operatorname{Dom}(U X)=U\{\operatorname{Dom}(M) ; M \in X\}$ and

$$
(U X)(K)=\sup \{M(K) ; M \in X \& K E M\} \text {, for } K \in \operatorname{Dom}(U X) \text {. }
$$

(b) We define $\cap X$ by $\operatorname{Dom}(\cap X)=\cap\{\operatorname{Dom}(M) ; M \in X\}$ and

$$
(\cap X)(K)=\min \{M(K) ; M \in X \& K E M\} \text {, for } K \in \operatorname{Dom}(n X) \text {. }
$$

If $M$ is an $m$-set, then we define $U M, \cap M$ to be $U(\operatorname{Dom}(M)), \cap(\operatorname{Dom}(M))$ respectively.

As observed by Knuth, we could alter our definition of "U" by replacing "sup" with "sum". There is no logical reason why one of these two possible definitions should be given preferential treatment over the other; we made our choice simply because we are at present interested in the set-theoretical nature of multisets, and the above definition seems to fit in best with our investigations.

DEFINITION 5. Let $M, N$ be m-sets with $M \subseteq_{m} N:$ we define the m-set $N^{\prime}-M$ as follows.

(1) $\operatorname{Dom}(N-M)=\{K \in \operatorname{Dom}(N) ; K \notin \operatorname{Dom}(M)$ or $K \in \operatorname{Dom}(M) \& M(K)<N(K)\}$.

(2) For $K \in \operatorname{Dom}(N-M)$,

$$
\begin{array}{rlrl}
(N-M)(K) & =N(K) & & \text { if } K \notin \operatorname{Dom}(M) \\
& =\min \{x ; C(x) \& N(K)=M(K)+x\} & \text { if } K \in \operatorname{Dom}(M) .
\end{array}
$$

As far as we can see, the above definition is the one that best reflects the requirements of those who have used complementation of multisets. It is not however a particularly attractive definition, and it does have a couple of rather disturbing consequences.

This completes our list of fundamental definitions: we now turn our attention to the task of illustrating some of their logical consequences. The following is not in any way intended as a systematic presentation of a theory of multisets: we are concerned with showing, by a sequence of examples, some of the resemblances and some of the differences between classical set theory and a theory of multisets based upon the foregoing definitions.

To start; we see that the Axiom of Extensionality fails - if $M, N$ are m-sets, then the implication $M=N \Rightarrow \forall K(K E M \Longleftrightarrow K E N)$ holds, but its converse fails. This is hardly surprising, for it is in the very concept 
of membership that the concept of a multiset differs from the concept of a set.

It is true however that for any two m-sets, $M, N$ we have $M=N \Leftrightarrow\left(M \subset_{m} N \& N \subset_{m} M\right)$. The forward direction is of course a theorem of the underlying logic; and the reverse direction follows from the simple observation that

$$
\left(M \subsetneq_{m} N \& N \subset_{m} M\right) \Rightarrow(\operatorname{Dom}(M)=\operatorname{Dom}(N) \& \forall K \in \operatorname{Dom}(M)(M(K)=N(K))) .
$$

Two of the cornerstones of classical set theory are the SchröderBernstein theorem, which says that if $X, Y$ are sets and $f: X \rightarrow Y$, $g: Y \rightarrow X$ are injections, then there is a bijection $h: X \rightarrow Y$; and the Cantor powerset theorem, which says that for any set $X$, there is an injection $X \rightarrow P(X)$ but no injection $P(X) \rightarrow X$.

Both these theorems are non-extendable in any significant sense. Take the Cantor theorem first. If $M$ is any $m$-set, then there exists an injection $M \rightarrow P(M)$ only if $M$ is an ordinary set. For if $f: M \rightarrow P(M)$ is injective, then by our definitions we must have $I \leq M(K) \leq P(M)(f(K))=1$ for each $K E M$.

To show that the Schröder-Bernstein theorem does not in general hold for $m$-sets, we define $M, N$ by taking $\operatorname{Dom}(M)=\operatorname{Dom}(N)=\omega$ (the set of natural numbers), and for each $n<\omega$, we put $M(n)=2 n$, $N(n)=2 n+1$. We now define injective m-maps $r: M \rightarrow N, s: N \rightarrow M$ by $r(n)=n, s(n)=n+1$. There is however no bijective m-map $t: M \rightarrow N$, because for any such $t$ we would have to have $M(n)=N(t(n))$ for each $n$, which is of course impossible.

The m-set operations of $U, \cap$ are reasonably well-behaved, since they are defined in a straightforward in terms of the cardinal operations min, sup, which themselves follow closely the behaviour of the classical $U, \cap$. We can for example show that for m-sets $U$ and $\cap$ are idempotent, associative, commutative, and distributive over finite collections. We cannot of course expect full distributivity over infinite collections, for we do not have it in the classical case. Classically we have

$$
\cap\left\{S_{x} ; x \in X\right\} \cup \cap\left\{T_{x} ; x \in X\right\} \subseteq \cap\left\{S_{x} \cup T_{x} ; x \in X\right\}
$$


and

$$
\cup\left\{S_{x} ; x \in X\right\} \cap \cup\left\{T_{x} ; x \in X\right\} \supseteq \cup\left\{S_{x} \cap T_{x} ; x \in X\right\},
$$

where $\left\{S_{x}{ }_{x \in X}, \quad\left\{{ }^{2}\right\}_{x \in X}\right.$ are families of ordinary sets; and these inclusions also hold for $m$-sets. In every case the method of proof consists in writing out the expression in terms of min and sup, and taking it from there.

The complementation operator is not so considerate. Consider $M=\{a .5, b .5\}, N=\{a .3, b .3\}$, where we have adopted the notational convention of expressing an $m$-set $R$ as $\{K . R(K) ; K \in \operatorname{Dom}(R)\}$. We have $\emptyset \neq N \varsubsetneqq_{m} M$, and yet $M-N=\{a .2, b .2\} \varsubsetneqq_{m} N$, conflicting with the classical law $(M-N) \cap N=\varnothing$.

Another case involves de Morgan's laws

$$
\left[M-U\left\{N_{x} ; x \in X\right\}=\cap\left\{M-N_{x} ; x \in X\right\} \text {, and its other half }\right] \text {. }
$$

If we take the iniex set $X$ to be finite, then de Morgan's laws do hold for $m$-sets. The proof of this consists quite simply in expressing each side in terms of min, sup, and performing some rather tedious manipulations.

When the index set is infinite however, the situation is quite different. Take $M=\left\{n . \aleph_{0}\right\}_{n<\omega}$; that is, $M$ consists of all the natural numbers, with each one being repeated $\aleph_{0}$ times. For each $n$ put $N_{n}=\{k \cdot n+1-k\}_{k \leq n}$. Thus $N_{0}=\{0.1\}, N_{1}=\{0.2,1.1\}$, $N_{2}=\{0.3,1.2,2.1\}$, and so on. Clearly $\cup\left\{N_{n} ; n<\omega\right\}=M$, and so $M-U\left\{N_{n} ; n<\omega\right\}=\emptyset$. On the other hand, $M-N_{n}=M$ for each $n$, whence $\cap\left\{M-N N_{n} ; n<\omega\right\}=M$.

We concede that this is a contrived example, and that in most of the cases arising in practice, de Morgan's laws would hold. We maintain, however, that this example, like the preceding ones, shows that if multisets are to play a role in mathematics, then their properties should not be taken lightly. 


\section{References}

[1] Nachum Dershowitz and Zohar Manna, "Proving termination with muitiset orderings", Automata, Languages and Programming, 188-202 (Proc. Sixth Colloquium, Graz, Austria, 1979. Lecture Notes in Computer Science 71. Springer-Verlag, Berlin, Heidelberg, New York, 1979.

[2] Donald E. Knuth, Seminumerical algorithms: the art of computer programming, Volume 2 (Addison Wesley, Reading, Massachusetts; Menlo Park, California; London; 1969).

Department of Mathematics,

Institute of Advanced Studies,

Australian National University,

PO Box 4,

Canberra, ACT 2600,

Australia. 